\title{
SÍNDROME DE BURNOUT Y ESTRATEGIAS DE AFRONTAMIENTO EN ACOMPAÑANTES TERAPÉUTICOS
}

\author{
BURNOUT SYNDROME AND COPING STRATEGIESAMONG THERAPEUTIC \\ ATTENDANTS \\ CECILIA REYNA ${ }^{1}$ \\ Facultad de Psicología de la Universidad Nacional de Córdova, Argentina \\ (RECIBIDO 08/08/2011, ACEPTADO 29/11/2011)
}

\begin{abstract}
RESUMEN
El objetivo central de este estudio fue analizar las manifestaciones de Síndrome de Burnout en un colectivo particular de agentes de salud, los acompañantes terapéuticos. Asimismo, se indagó acerca de las estrategias de afrontamiento empleadas, tanto en términos generales como específicas de la profesión, y su relación con los síntomas del síndrome. La muestra estuvo compuesta por 17 profesionales de Córdoba (Argentina) de ambos sexos (41.2\% varones), con edades comprendidas entre los 22 y 53 años $(M=29.53$ años, $D T=7.43)$. La prevalencia de los distintos síntomas del síndrome se examinó considerando distintos criterios de corte, con resultados dispares; mientras que las relaciones con las estrategias de afrontamiento sólo se manifestaron entre algunas dimensiones.
\end{abstract}

Palabras clave: Síndrome de Burnout; afrontamiento; trabajadores de la salud

\begin{abstract}
The purpose ofthisstudywasto analyzethe manifestations ofBurnout syndrome inaparticular groupofhealth workers, therapeuticattendants.In addition,coping strategiesarestudied, both in generaltermsandspecificstrategiesoftheprofession, anditsrelationshipwithsymptomsof the syndrome. The sample consisted of 17 professionals from Córdoba(Argentina) of both sexes (41.2\% males), agedbetween22 and53 years $(M=29.53$ years, $S D=7.43)$. The prevalenceof different symptomsof the syndromeisdiscussedconsideringdifferentcriteriaof cut, withmixed results; while relations withthe coping strategieswere only evident among some dimension.
\end{abstract} Keywords: Burnout Syndrome; coping; healthworkers

1 Docente de la Facultad de Psicología, Universidad Nacional de Córdova, Ciudad Universitaria, Argentina, E-maill: ceciliareyna@gmail.com 


\section{INTRODUCCIÓN}

Desde una perspectiva psicosocial el Síndrome de Burnout (SBOO en adelante) se conceptualiza como una respuesta al estrés laboral crónico que se caracteriza por pensamientos de fracaso profesional, vivencia de agotamiento emocional y actitudes negativas hacia las personas con las que se trabaja (Gil-Monte y Peiró, 1999). Maslach y Jackson (1997) integran estos síntomas como dimensiones del Inventario de Burnout de Maslach, las cuales se denominan: baja realización personal en el trabajo, agotamiento emocional y despersonalización, respectivamente.

La baja realización personal en el trabajo hace referencia a la tendencia de los profesionales a evaluarse negativamente, esa valoración afecta la tarea del trabajo y la relación con las personas que se atienden. Los trabajadores se sienten descontentos consigo mismos e insatisfechos con sus resultados laborales.El agotamiento emocional alude a la situación en la que los trabajadores sienten que ya no pueden brindar más de sí mismos a nivel afectivo, es una situación de agotamiento de la energía o los recursos emocionales propios, una experiencia de estar emocionalmente agotado debido al contacto cotidiano con personas a las que hay que atender como objeto de trabajo. En tanto que la despersonalización se define como el desarrollo de sentimientos negativos, de actitudes y conductas de cinismo hacia las personas destinatarias del trabajo. Estas personas son vistas por los profesionales de manera deshumanizada debido a un endurecimiento afectivo (Maslach y Jackson, 1997).

El Síndrome de Burnout es el resultado de la exposición continua a estresores existentes en las áreas laborales, lo cual ha dado lugar a que diversas variables extra-laborales y laborales, tales como edad, sexo, estado civil, número de hijos, tipo de profesión, puesto laboral, tecnología utilizada, desempeño de roles, clima laboral, tipo de personalidad y apoyo social, hayan sido consideradas en mayor o menor grado como antecedentes o facilitadores del SBOO (Aranda, Pando y Pérez, 2004). Por su parte, Gil-Monte y Peiró (1997) resaltan los siguientes factores como desencadenantes principales del síndrome: ambiente físico de trabajo y contenidos del puesto; estrés por desempeño de roles, relaciones interpersonales y desarrollo de la carrera; estresores relacionados con las nuevas tecnologías y otros aspectos organizacionales y extra-organizacionales de estrés laboral.

Síndrome de Burnout en agentes de la salud

Con frecuencia el SBOOse manifiesta en los profesionales de la salud y, en general, en profesionales de organizaciones de servicios que trabajan en contacto directo con los usuarios de la organización. En el contexto de las organizaciones sanitarias son estresores especialmente relevantes para el desarrollo del síndrome las relaciones sociales de los profesionales de la salud con los pacientes y sus familiares. El SBOO resulta un tipo particular de mecanismo de afrontamiento y autoprotección frente al estrés generado por la relación profesional-clientey por la relación profesional-organización (Gil-Monte, 2001).Las situaciones laborales estresantes suscitan habitualmente una serie de estrategias de afrontamiento que, en el caso de los profesionales cuyo objeto de trabajo son personas, deben ser 
efectivas para manejar las respuestas al estrés, pero también han de ser eficaces para eliminar las situaciones estresantes. Cuando las estrategias de afrontamiento empleadas no resultan exitosas de manera reiterada, los trabajadores desarrollan una experiencia de fracaso profesional y de fracaso en las relaciones interpersonales con los individuos hacia los que se destina el trabajo (Gil-Monte, 2008).

García (1991, citado en Peinado y Garcés, 1998) remarca que el SBOO es un problema característico de los trabajos que implican servicios humanos, aquellas profesiones que deben mantener una relación continua de ayuda hacia el cliente, como médicos, profesores, enfermeras, asistentes sociales, psiquiatras, psicólogos, policías, entre otros. El objetivo de este tipo de profesiones es cuidar los intereses o satisfacer las necesidades del usuario, caracterizándose por el trabajo en contacto directo con los destinatarios. Por dicha razón, una de los síntomas del síndrome es el desgaste emocional que se va generando en el trabajador debido a la interacción con otros. El estrés en estas profesiones está compuesto por una combinación de variables físicas, psicológicas y sociales (Gil-Monte yPeiró, 1997).

En el contexto terapéutico, uno de los profesionales que pueden estar implicados es el acompañante terapéutico, quienes se encuentran expuestos a algunos de los estresores desencadenantes del SBOO, y en tal sentido, resulta relevante indagar acerca de la manifestación o no del síndrome en estos profesionales.

El acompañamiento terapéutico comienza como una necesidad del terapeuta, quien designa a una persona entrenada y capacitada para la contención. Su presencia en sí es un acto terapéutico, acto a través del cual se establece un vínculo que la persona no tuvo hasta ese momento, y que le posibilitará instaurar una diferencia, creando nuevos lazos de resocialización. Acompañar es estar con el otro, compartir. Hay una estrategia dirigida a una cura, y esto es lo que la diferencia de una situación no terapéutica, en la que sólo se comparte algo con el otro. Lo curativo hace que 'desaparezcan' los síntomas, haya un enriquecimiento personal y se adquiera la tan ansiada capacidad o habilidad para estar solo (Cavagna, 1996).

Las funciones principales de los acompañantes terapéuticos, según mencionan Kuras y Resniki (2004), son: contener al paciente, ofrecerse como referente, ayudar a 'reinvestir' (se ofrece a la manera de un organizador psíquico), registrar y ayudar a desplegar la capacidad creativa del paciente, aportar una mirada ampliada del mundo objetivo del paciente, habilitar un espacio para pensar, orientar en el espacio social, e intervenir en la trama familiar. Además, los autores señalan que los acompañantes terapéuticos pueden trabajar con personas en distintas condiciones, como por ejemplo, ambulatorios, asistentes a hospital de día u hospital de noche, con internación completa, con atención domiciliaria, o en situación de convivencia con familia sustituta.

El concepto de SBOO se relaciona íntimamente con el concepto de riesgos de equipo y autocuidado. Este concepto surge de la práctica de supervisión clínica, particularmente desde la perspectiva teórica psicoanalítica y sistémica contextual, intentando describir, interpretar y proponer modalidades de intervención que promuevan el autocuidado y disminuyan los riesgos de equipo. En este sentido, 
Morales, Pérez y Menares (2003) investigaron el proceso de desgaste emocional en personas cuyo trabajo se relacionaba con el sufrimiento y daño social de niños y jóvenes, y observaron que el criterio de autocuidado no estaba establecido, era difuso, aunque se reconocían los propios límites en la acción profesional, y la necesidad de establecer límites entre los ámbitos laboral y familiar. Además, encontraron que la duración del vínculo de los profesionales con los usuarios interfería en el curso de los procesos emocionales.

Gil-Monte, Carretero, Roldán y Román (2005) analizaron la prevalencia del SBOO en monitores y educadores de centros para personas discapacitadas, utilizando el Cuestionario para la Evaluación del Síndrome de Quemarse por el Trabajo en su versión para profesionales que trabajan con discapacitados (CESQT-PD) y escalas del Inventario de Burnout de Maslach (realización personal en el trabajo y despersonalización del MBI-HSS, y agotamiento del MBI-General Survey). Los resultados muestran que el SBOO afectaba al $11.70 \%$ de los participantes,y si bien no se observaron diferencias significativas de género, las mujeres representaban un alto porcentaje en la categoría más alta del CESQT-PD. En otro de los trabajos realizados por el equipo de Gil-Monte (Olivarez y Gil-Monte, 2007) se empleó el CESQT-PD para evaluar la prevalencia del SBOO en profesionales de servicios humanos en Chile, observándose que de 393 participantes, el 5.09\% presentaba bajas puntuaciones en las dimensiones de ilusión por el trabajo y altas puntuaciones en desgaste psíquico e indolencia, aunque sólo el $1.27 \%$ fueron casos graves, con altas puntuaciones en el las dimensiones mencionadas y además altos sentimientos de culpa. Por otra parte, estudios realizados con personal médico de las provincias de Rosario y Santa Fe en Argentina (Sosa, 2007) señalan que el $76 \%$ de la muestra analizada presentaba cuatro o más síntomas del SBOO, sin diferencias significativas al considerar variables tales como sexo, edad, especialidad médica, años de ejercicio de la profesión, ámbito de trabajo, horas de trabajo por semana y cantidad de horas de guardia médica

Diversos investigadores han analizado las implicancias del ambiente de trabajo en el SBO (Robayo y Torres, 2002). Topa y Morales (2007) investigaron la relación del SBOcon las identificaciones dentro del grupo, y observaron que los participantes (empleados de distintos grupos de trabajo de varias organizaciones) que se identificaban más con su grupo presentaban menores niveles de SBO; al mismo tiempo, encontraron que el apoyo social mediaba parcialmente las relaciones entre la identificación grupal y el cansancio emocional.

Por otra parte, Boada, Vallejo y Agulló (2004) investigaron si las variablesimplicadas en el modelo de las características del puesto son predictoras de los niveles deSBOy de síntomas psicosomáticos en el trabajo. Dicho modelo permite determinar las condiciones laborales que generan una alta motivación interna en los trabajadores, comprendiendo las siguientes variables moduladoras: capacidades y conocimientos relevantes para el puesto de trabajo, intensidad de la necesidad de autorrealización y satisfacción con los factores del contexto de trabajo (supervisor, compañeros, remuneración y seguridad en el trabajo). Los investigadores observaron que 
las variables del modelo analizado cumplían un rol preventivo y predictor en relación a las distintas dimensiones del SBO, pero no respecto de los síntomas psicosomáticos estudiados.

Con respecto a la relación entre el SBO y variables sociodemográficas, Atance (1997) resume diversas investigaciones de la siguiente manera: a) la edad parece no influir, aunque se reconoce un período sensible correspondiente a los primeros años de ejercicio profesional en donde se pasaría de las expectativas idealistas a la práctica cotidiana; b) las mujeres serían más vulnerables a manifestar SBO, lo cual está en línea con lo planteado por Párraga (2005), pero no con las observaciones de otros investigadores que han observado que los hombres presentan más cansancio emocional que las mujeres (Caballero, Bermejo, Nieto y Caballero, 2001); c) se presenta más en personas que no tienen una pareja estable, aunque no hay un claro consenso respecto de este punto; d) las personas con hijos mostrarían mayor flexibilidad para manejar situaciones estresantes; e) el cambio horario, la sobrecarga laboral y bajas remuneraciones se relacionan de manera positiva con las manifestaciones del SBO.

\section{Estrategias de afrontamiento y Síndrome de Burnout}

La conducta de afrontamiento domina las demandas de situaciones de estrés y puede implicar una actitudactiva tendiente a controlar la situación o una actitud pasiva ante la misma (Guerrero, 2003). El grado en que los recursos por sí mismos neutralizan los efectos del estrés en comparación con los procesos reales de afrontamiento fue demostrado empíricamente por Pearlin y Schooler en 1984, distinguiendo los recursos que son propiedad del individuo,como la salud y la energía (recursos físicos), las creencias positivas (recursos psicológicos), las técnicas de resolución de problemas, el apoyo social, y los recursos ambientales (recursos sociales y materiales). Estos recursos, como señalan Lazarus y Folkman (1986), son los que determinan el proceso de afrontamiento. Cabe diferenciar los estilos de afrontamiento y las estrategias de afrontamiento. Los primeros se refieren a predisposiciones personales para hacer frente a las situaciones y son responsables de las preferencias individuales en el uso de unos u otros tipos de estrategia de afrontamiento, así como de su estabilidad temporal y situacional; mientras que las segundas son los procesos concretos que se utilizan en cada contexto y son altamente cambiantes dependiendo de las situaciones desencadenantes (FernándezAbascal, 1998).

Lazarus y Folkman (1986) definen el afrontamiento como los esfuerzos cognitivos y conductuales constantemente cambiantes que se desarrollan para controlar las demandas específicas externas y/o internas que son evaluadas como excedentes o desbordantes de los recursos del individuo.El afrontamiento es una estrategia de marcado carácter individual, modulando por ello el carácter estresante o no de las situaciones, e implica las observaciones y valoraciones que el individuo realmente piensa o hace analizadas dentro de un contexto específico y dirigido hacia condiciones particulares. Es un cambio en los pensamientos y actos a medida que la interacción se desarrolla, ya que el sujeto mantiene continuas evaluaciones 
y reevaluaciones de la cambiante relación individuo-entorno, siendo indispensable para su comprensión una visión dinámica del fenómeno. En términos generales, se considera que existen dos grandes categorías de estrategias para afrontar el estrés: 1) afrontamiento directo o acción directa, estrategia aplicada externamente a la fuente ambiental de estrés para dominar la transacción estresante con el ambiente, se dirige a la definición del problema, a la búsqueda de soluciones alternativasy a su aplicación, generalmente se utiliza cuando las condiciones evaluadas resultan como susceptibles de cambio; y2) afrontamiento indirecto o acción paliativa, estrategia aplicada a las propias emociones, se intenta reducir el trastorno cuando se es incapaz de controlar el ambiente o la acción es demasiado costosa (Hernández, Olmedo e Ibáñez, 2004).Por otra parte, otros autores identifican un tercer tipo de afrontamiento, el apoyo social, mientras que otros lo consideran como una modalidad intermedia debido a que incluye componentes de los dos tipos generales o lo clasifican como una estrategia focalizada en el problema (Parris, 2007).

Popp (2008) realizó un estudio en Argentina sobre el SBO y las estrategias de afrontamiento en personal de enfermería aplicando el Inventario Burnout de Maslach y el Inventario de Modos de Afrontamiento de Lazarus y Folkman, concluyendo que existen diferencias significativas en las estrategias utilizadas entre las enfermeras que presentan diferentes niveles de SBO, principalmente en cuanto a las dimensiones de despersonalización y baja realización personal. Las enfermeras utilizaban estrategias de afrontamiento menos funcionales a medida que aumentaba el nivel de SBO, quienes presentaban niveles menores de realización personal utilizaban menos análisis lógicos, gratificaciones alternativas, ejecución de acciones que conduzcan a la resolución del problema. Las enfermeras con un nivel medio de cansancio emocional empleaban más el control afectivo en relación a quienes tenían un nivel bajo o alto de agotamiento emocional. Sin embargo, las relaciones entre estrategias de afrontamiento y SBO no resultan totalmente claras aún; por ejemplo, Zamora, Castejón y Fernández (2003) sólo encontraron relaciones significativas entre el factor evitación-escape de las estrategias de afrontamiento y la puntuación general de SBO, y entre el factor distanciamiento y la dimensión despersonalización del SBO.

La relación entre estos constructos ha sido también analizada con otros profesionales, como por ejemplo, docentes (Guerrero, 2003), observando que los profesores con altos niveles de SBO empleaban estrategias tales como desconexión conductual y consumo de drogas, y por el contrario, quienes manifestaban niveles elevados de realización personal empleaban más estrategias de afrontamiento activas, tales como planificación, búsqueda de apoyo instrumental, búsqueda de apoyo social y reinterpretación positiva.

Los diversos estudios revisados coinciden en la manifestación del SBO en agentes de la salud que mantienen un contacto directo con los pacientes. Sin embargo, no se encontraron investigaciones puntuales en acompañantes terapéuticos, y menos aún que indaguen la relación entre el SBO y las estrategias de afrontamiento. De esta manera, el presente estudio se propone examinar las posibles manifestaciones de 
SBO en acompañantes terapéuticos de Córdoba (Argentina) considerando distintos criterios de corte, y además, analizar la relación con las estrategias de afrontamiento empleadas, y las diferencias en el SBOy en las estrategias de afrontamiento al considerar variables sociodemográficas y laborales.

\section{MÉTODO}

\section{Participantes}

La muestra accidental estuvo conformada por 17 acompañantes terapéuticos de la ciudad de Córdoba (Argentina) que trabajan como tal al momento de desarrollo del estudio, convocados a través de dos organizaciones de la ciudad.

\section{Instrumentos}

Cuestionario de datos sociodemográficos y laborales. A través de este cuestionario se indagaron los siguientes aspectos: sexo, edad, estado civil, hijos, otras profesiones, otras carreras en estudio, cantidad de trabajo como acompañante terapéutico, tipo y edades de los pacientes, y desempeño en otras actividades.

Inventario de Burnout de Maslach. Se utilizó la versión HumanSercicesSurvey por ser el instrumento más utilizado para evaluar SBOen personas que desempeñan actividades asistenciales. El inventario comprende tres dimensiones: agotamiento emocional (sensación de no poder dar más de sí en el ámbito emocional y afectivo), despersonalización (desarrollo de sentimientos de insensibilidad y actitudes negativas hacia los destinatarios de la labor profesional) y realización profesional (niveles bajos se asocian al SBO, con sentimientos de improductividad o falta de logros en el desempeño profesional). Está compuesto por 22 ítems en forma de afirmaciones sobre los sentimientos y actitudes del profesional en su trabajo, y hacia los clientes o usuarios. La valoración de cada ítem se realiza a través de una escala tipo Likert de 7 puntos, donde $0=$ Nunca y $6=$ Diariamente. La puntuación máxima para la escala agotamiento emocional es 54 , para despersonalización es 30 y para realización personal es 48 . A pesar de su extenso uso, los criterios de corte empleados en distintos estudios para la determinación de los niveles de SBO no siempre coinciden. Gil-Monte y Marucco (2008) recuperan criterios de corte obtenidos con muestra estadounidense propuestos por Maslach y Jackson (1986), con muestra española por Gil-Monte y Peiró (2000), y con muestra argentina en el estudio de Neira (2004), y para establecer criterios patológicos recurren a Schaufeli y Dierendock (1995). En este trabajo se consideran los tres primeros criterios y además, la puntuación a partir de percentiles.

Estrategias de Afrontamiento. Se empleó el Inventario de Estrategias de Afrontamiento de Tobin, Horoyd, Reynolds y Kigal en su versión española traducida por Cano, Rodríguez y García (2007). Este inventario presenta una estructura jerárquica compuestapor ocho estrategias primarias, cuatro secundarias y dosterciarias.Las escalas primarias son: resolución de problemas (estrategias cognitivas y conductuales encaminadas a eliminar el estrés modificando la situación que lo produce), reestructuración cognitiva (estrategias cognitivas que 
modifican el significado de la situación estresante), apoyo social (estrategias referidas a la búsqueda de apoyo emocional), expresión emocional (estrategias encaminadas a liberar las emociones que acontecen en el proceso de estrés), evitación de problemas (estrategias que incluyen la negación y evitación de pensamientos o actos relacionados con el acontecimiento estresante), pensamiento desiderativo (estrategias cognitivas que reflejan el deseo de que la realidad no fuera estresante), retirada social (estrategias de retirada de amigos, familiares, compañeros y personas significativas asociada con la reacción emocional en el proceso estresante), y autocrítica (estrategias basadas en la autoinculpación y la autocrítica por la ocurrencia de la situación estresante o su inadecuado manejo). Las escalas secundarias surgen de la agrupación de las primarias: manejo adecuado centrado en el problema (resolución de problemas y reestructuración cognitiva), manejo adecuado centrado en la emoción (apoyo social y expresión emocional), manejo inadecuadocentrado en el problema (evitaciónde problemas y pensamiento desiderativo), y manejoinadecuado centrado en la emoción(retiradasocial y autocrítica). Finalmente, las escalas secundarias se agrupan dando lugar a dos grandes categorías: manejo adecuado y manejo inadecuado. Además, se realizaron otras preguntas con el objetivo de indagar acerca de posibles estrategias de afrontamiento que pueden emplear los acompañantes terapéuticos y que no eran contempladas en el inventario mencionado. Específicamente, los ítems añadidos fueron: "Consulté o hablé con el profesional a cargo", "Dejé que el profesional a cargo me ayudase", "Le pedí al profesional a cargo un consejo para saber qué hacer", "Me apoyé en el equipo terapéutico", y "Supervisé mi tarea".

\section{Procedimiento}

En primera instancia se solicitó la colaboración de dos organizaciones donde trabajaban acompañantes terapéuticos y luego se tomó contacto con los mismos, explicando los objetivos del estudio y el carácter confidencial y voluntario de su participación. Los participantes completaron los distintos instrumentos en formato papel o vía electrónica. Luego de la recolección de datos, los mismos fueron analizados con el paquete estadístico SPSS 17.

\section{RESULTADOS}

\section{Características descriptivas}

En primer lugar se describen las características sociodemográficas y laborales. El $41.2 \%$ de los participantes eran varones y el $58.8 \%$ mujeres, con edades comprendidas entre los 22 y 53 años $(M=29.53$ años, $D T=7.43)$. El $64.7 \%$ manifestó estar soltero, el $17.6 \%$ casado y el $17.6 \%$ conviviendo, mientras que sólo el $11.8 \%$ informó tener hijos.En cuanto a aspectos laborales, el $29.4 \%$ trabaja sólo como acompañante terapéutico (AT), el $41.2 \%$ trabaja como AT y estudia, y el $29.4 \%$ trabaja como AT y en otra actividad. La media de horas trabajadas por semana como AT es de $12.41(D T=7.15)$, el $58.8 \%$ trabaja hasta 14 horas y el $41.2 \% 15$ horas o más. El $41.2 \%$ realiza sólo un acompañamiento actualmente y el 58.8\% 2 o más acompañamientos. Con respecto a los pacientes, el $70.6 \%$ señala 
trabajar en el área de salud mental, el $47.1 \%$ en discapacidad y el $11.8 \%$ en el área judicial; mientras que $35.3 \%$ trabaja con niños, el $41.2 \%$ con adolescentes, el $76.5 \%$ con adultos y el $5.9 \%$ con ancianos.

Síndrome de Burnout según distintos puntos de corte

Se calcularon estadísticos descriptivos a partir de las puntuaciones brutas. En Agotamiento Emocional (AE) la media fue de 16.82 ( $D T=9.86$, Mediana $=19)$, en Despersonalización $(\mathrm{D})$ fue $3.06(D T=3.60$, Mediana $=3)$ y en Realización Personal en el Trabajo $(\mathrm{RP})$ fue $41.06(D T=5.03$, Mediana $=43)$.

En la Tabla 1 y Figuras 1 a 3 se muestra el porcentaje de participantes en las categorías bajo, medio y alto de cada dimensión según distintos criterios de corte. Los niveles de Agotamiento Emocional más altos se manifiestan siguiendo los criterios de Neira (2004), y luego acorde a los criterios de Gil-Monte y Peiró (2000), los valores a partir de percentiles y por último el criterio de Maslach y Jackson (1986). Los criterios para las puntuaciones más bajas son semejantes según Gil-Monte y Peiró (2000) y Maslach y Jackson (1986).

En cuanto a Despersonalización, los criterios de Neira (2004) y los percentiles dan lugar a proporciones semejantes, y más altas que las de Gil-Monte y Peiró (2000) y Maslach y Jackson (1986), según este último criterio ningún participante manifiesta niveles altos de D.

En Realización Personal en el Trabajo, según los criterios de Neira (2004) y Gil-Monte y Peiró (2000) las proporciones son semejantes, un poco más altas en relación a los criterios de Maslach y Jackson (1986) en la categoría bajo. En esta dimensión los valores en percentiles dan lugar a proporciones bastante distintas al resto de los criterios.

Comúnmente se considera que el SBOestá presente si las puntuaciones son altas en AE, D y bajas en RP. Sin embargo, en la literatura se señala que no necesariamente se deben combinar las dimensiones para identificar el síndrome (Briones, 2007), mientras que algunos autores han propuesto agrupación de categorías (Cáceres, 2006). A partir de ello, con el objetivo de identificar aquellas personas que presentaban SBOse agruparon las categorías medio y alto (alto-b) de AE y D, y bajo y medio de RP (bajo-b), quedando sólo dos categorías en cada dimensión. La presencia del síndrome se determinó a partir de puntuaciones altas-b en AE y D y bajas-b en RP. En la Figura 4 se muestra la prevalencia del síndrome según los distintos criterios de corte. Según los criterios de Maslach y Jackson (1986) y Gil-Monte y Peiró (2000) el 5.9\% de los participantes presentan SBO; según el criterio de Neira (2004) el 17.6\%; y según los valores en percentiles el 52.9\% presenta el síndrome. 
Tabla 1. Síndrome de Burnout según distintos puntos de corte

\begin{tabular}{|c|c|c|c|c|c|c|c|c|c|c|c|c|}
\hline \multirow{2}{*}{$\begin{array}{l}\text { Puntos } \\
\text { de Corte }\end{array}$} & \multicolumn{3}{|c|}{$\begin{array}{c}\text { Maslach y Jackson } \\
\text { (1986) }\end{array}$} & \multicolumn{3}{|c|}{$\begin{array}{c}\text { Gil-Monte y Peiró } \\
(2000)\end{array}$} & \multicolumn{3}{|c|}{ Neira (2004) } & \multicolumn{3}{|c|}{ Percentiles } \\
\hline & $\mathrm{AE}$ & D & $\mathrm{RP}$ & $\mathrm{AE}$ & D & $\mathrm{RP}$ & $\mathrm{AE}$ & $\mathrm{D}$ & $\mathrm{RP}$ & $\mathrm{AE}$ & D & $\mathrm{RP}$ \\
\hline Bajo & $\leq 16$ & $\leq 6$ & $\leq 31$ & $\leq 15$ & $\leq 3$ & $\leq 35$ & $\leq 11$ & $\leq 2$ & $\leq 35$ & $\leq 25$ & $\leq 25$ & $\leq 25$ \\
\hline$\%$ & 47.1 & 82.4 & 5.9 & 47.1 & 76.5 & 17.6 & 35.3 & 47.1 & 17.6 & 23.5 & 35.3 & 23.5 \\
\hline Medio & $26-17$ & $12-7$ & $38-32$ & $24-16$ & $8-4$ & $39-36$ & $12-21$ & $3-6$ & $36-40$ & $26-75$ & $26-75$ & $26-75$ \\
\hline$\%$ & 41.2 & 17.6 & 17.6 & 23.5 & 11.8 & 5.9 & 17.6 & 35.3 & 11.8 & 52.9 & 41.2 & 52.9 \\
\hline Alto & $\geq 27$ & $\geq 13$ & $\geq 39$ & $\geq 25$ & $\geq 9$ & $\geq 40$ & $\geq 22$ & $\geq 5$ & $\geq 41$ & $\geq 76$ & $\geq 76$ & $\geq 76$ \\
\hline$\%$ & 11.8 & - & 76.5 & 29.4 & 11.8 & 76.5 & 47.1 & 17.6 & 70.6 & 23.5 & 23.5 & 23.5 \\
\hline
\end{tabular}

AE: agotamiento emocional, D: despersonalización, RP: realización personal en el trabajo

Figura 1. Agotamiento Emocional según distintos puntos de corte

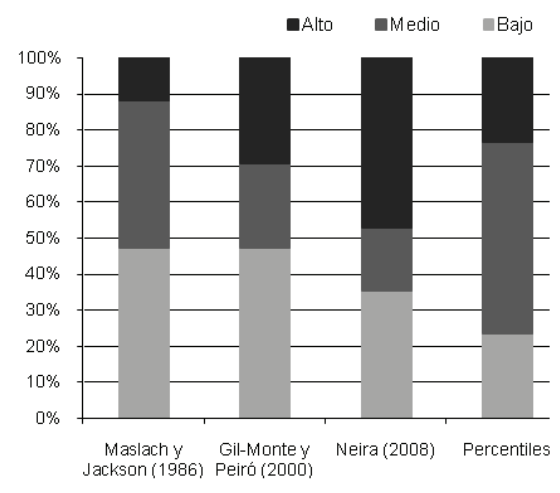

Figura 2. Despersonalización según distintos puntos de corte

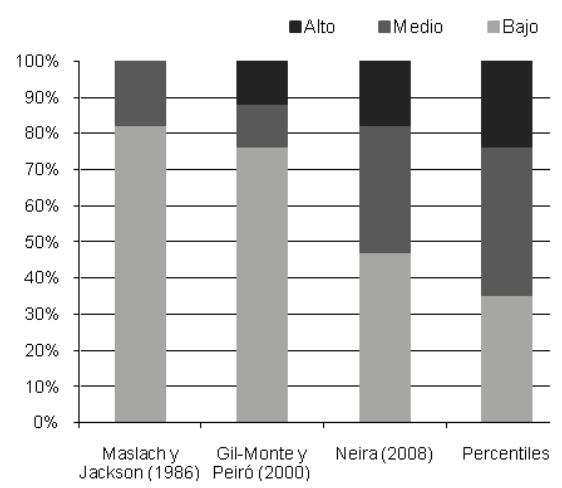


Figura 3. Realización Personal en el Trabajo según distintos puntos de corte

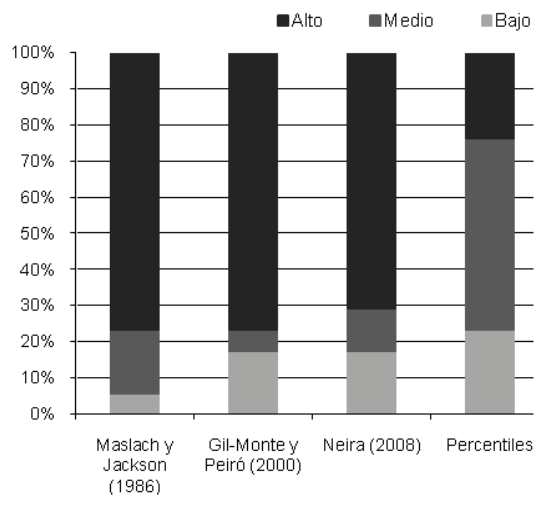

Figura 4. Prevalencia del Síndrome de Burnout según distintos puntos de corte

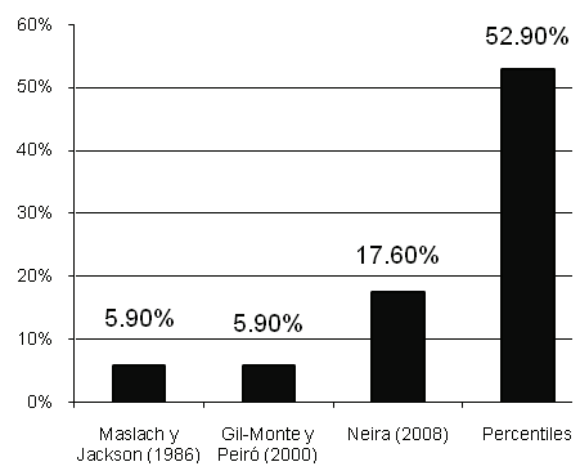

Relación con variables sociodemográficas y laborales

Se examinó la relación entre las categorías de las dimensiones del SBOOsiguiendo el criterio de Neira (2004) y variables sociodemográficas y laborales, sin observarse asociaciones significativas. Tampoco se observaron asociaciones estadísticamente significativas con las escalas de Estrategias de Afrontamiento.

Junto con el Cuestionario de Estrategias de Afrontamiento se realizaron preguntas que apuntaban a estrategias para afrontar problemas en el contexto de trabajo de un AT. Como se puede apreciar en la Tabla 2, gran parte de los participantes manifiesta recurrir al profesional a cargo, al equipo terapéutico y a supervisión para afrontar situaciones de estrés. 
Tabla 2. Porcentajes de respuesta en preguntas de afrontamiento de AT

\begin{tabular}{|c|c|c|c|c|c|}
\hline & En absoluto & Un poco & Bastante & Mucho & Totalmente \\
\hline $\begin{array}{l}\text { - Consulté o hablé } \\
\text { con el profesional a } \\
\text { cargo. }\end{array}$ & - & - & 29.4 & 29.4 & 41.2 \\
\hline $\begin{array}{l}\text { - Dejé que el } \\
\text { profesional a cargo } \\
\text { me ayudase. }\end{array}$ & - & 5.9 & 29.4 & 23.5 & 41.2 \\
\hline $\begin{array}{l}\text { - Le pedí a l } \\
\text { profesional a cargo } \\
\text { un consejo para saber } \\
\text { qué hacer. }\end{array}$ & - & 11.8 & 17.6 & 29.4 & 41.2 \\
\hline $\begin{array}{l}\text { - Me apoyé en el } \\
\text { equipo terapéutico. }\end{array}$ & - & - & 23.5 & 23.5 & 52.9 \\
\hline - Supervisé mi tarea. & - & - & 23.5 & 17.6 & 58.8 \\
\hline
\end{tabular}

\section{Relación entre Síndrome de Burnout y Estrategias de Afrontamiento}

Para analizar la relación entre las variables se consideraron las puntuaciones brutas de las escalas que evalúan SBOOy estrategias de afrontamiento. Debido al tamaño muestral se recurrió a estadísticos no paramétricos, en este caso el coeficiente de correlación de Spearman. Reestructuración Cognitiva se relacionó positivamente con RP $\left(r_{s}=0.54, p=0.025\right)$, esto es, mientras mayores estrategias de reestructuración cognitiva se utilizan para afrontar situaciones de estrés, mayor es la realización personal en el trabajo. Asimismo, la estrategia de Apoyo Social también mostró una relación positiva con RP $\left(r_{s}=0.623, p=0.008\right)$. Finalmente, Resolución de Problemas sólo mostró relaciones marginalmente significativas con $\mathrm{AE}\left(r_{s}=-0.468, p=0.058\right)$ y $\mathrm{RP}\left(r_{s}=0.473, p=0.055\right)$, lo cual significa que los participantes con mayor capacidad de resolución de problemas presentan menos agotamiento emocional y más realización personal en el trabajo. El resto de las variables no mostraron relaciones estadísticamente significativas.

\section{DISCUSIÓN}

La escasa investigación sobre la prevalencia del SBOO en Argentina y casi nula indagación en acompañantes terapéuticos impidió contar con puntos de cortes confiables y antecedentes para tomar como referencia. Por dicha razón se presentaron tres puntos de cortes a fin de evitar llegar a conclusiones equivocadas que podrían aparecer por utilizar un punto de corte que no concuerde con el valor o característica cultural laboral de la muestra en estudio. Sería necesario recurrir a otras técnicas, como por ejemplo entrevistas, para confirmar o descartar los casos identificados con SBOO.

Si bien no se encontraron relaciones significativas entre el sexo, la edad, estado civil, horas por semana trabajadas y cantidad de acompañamiento respecto al 
SBOO y las estrategias de afrontamiento, es posible que ello se deba al reducido tamaño muestral. Probablemente, una muestra de mayor tamaño ofrezca mayor heterogeneidad y así permita examinar más en detalle las relaciones estas variables.

Las relaciones encontradas entre las variables de SBOO y afrontamiento concuerdan con el trabajo de Popp (2008) realizado con enfermerasen Argentina, en el que se observa la relación positiva entre la ejecución de acciones tendientes a resolver el problema y la realización personal, aunque en este estudio las relaciones fueron marginalmente significativas.La ejecución de tareas se trata de una estrategia funcional respecto al SBOO, su uso disminuye los niveles de algunas de las dimensiones del síndrome. La relación positiva entre reestructuración cognitiva y apoyo social respecto de la realización personal coinciden con el trabajo de Guerrero (2003), donde se destaca la reinterpretación positiva dentro de la reestructuración cognitiva, con mayores niveles de realización personal asociados a mayor planificación, búsqueda de apoyo instrumental y búsqueda de apoyo social, .

Finalmente, la alta tasa de respuesta en relación a los ítems añadidos al cuestionario de estrategias de afrontamiento parece reflejar la necesidad de indagar estrategias de afrontamiento específicas de cada actor y/o contexto, lo cual se debería tener en cuenta en estudios futuros.

Fruto de este estudio, se subraya la necesidad de comprender en la literatura sobre SBOO a estos nuevos agentes de la salud, para luego de comprender sus manifestaciones específicas generar acciones tendientes a la prevención y reforzamiento de adecuadas estrategias de afrontamiento ante las situaciones de estrés que se manifiestan de manera cotidiana en su trabajo.

\section{REFERENCIAS BIBLIOGRAFICAS}

Aranda, A., Pando, M. y Pérez, M. (2004). Apoyo social y Síndrome de quemarse en el trabajo o Burnout: Una revisión. En Psicología y Salud, 14, pp. 79-87.

Atance, J. (1997). Aspectos epidemiológicos del Síndrome de Burnout en personal sanitario. En Revista Española de Salud Pública, 71, pp. 293-303.

Boada, J., Vallejo, R. y Agulló, E. (2004). El Burnout y las manifestaciones psicosomáticas como consecuentes del clima organizacional y de la motivación laboral. En Psicothema, 16, pp. 125-131.

Briones, D. (2007). Presencia de Síndrome de Burnout en poblaciones policiales vulnerables de carabineros de Chile. En Ciencia y Trabajo, 9, pp. 43-50.

Caballero, M., Bermejo, R., Nieto, R. y Caballero, F. (2001). Prevalencia y factores asociados al Burnout en un área de salud. En Atención primaria, 27, pp. 53-59.

Cano, F., Rodríguez, L. y García, J. (2007). Adaptación española del Inventario de Estrategias de Afrontamiento. En Actas Españolas de Psiquiatría, 35, pp. 29-39.

Cavagna, N. (1996).¿Qué es el Acompañamiento terapéutico? En Revista de Psiquiatría Dinámica y Psicología Clínica, 1, pp. 73-80. 
Fernández-Abascal, E. G. (1998). Estilos y estrategias de afrontamiento. En E.G. Fernández-Abascal, F. Palmero, M. Chóliz y F. Martínez. Cuaderno de prácticas de motivación y emoción, Madrid: Pirámide, pp. 189-206.

Gil-Monte, P. R. (2001). El síndrome de quemarse por el trabajo (Síndrome de Burnout): aproximaciones teóricas para su explicación y recomendaciones para la intervención. En Revista Electrónica Psicología Científica. Trabajo publicado 25 de julio. Recuperado el 20 de noviembre de 2009 de http://www. psicologiacientifica.com.

Gil-Monte, P. R. (2008). Maldito Trabajo, Barcelona: Granica.

Gil-Monte, P. R., Carretero, N., Roldán, M. y Núñez, E. (2005). Prevalencia del síndrome de quemarse por el trabajo (Burnout) en monitores de taller para personas con discapacidad. En Revista de Psicología del Trabajo y de las Organizaciones, 21, pp. 107-123.

Gil-Monte, P. R. y Marucco, M. (2008). Prevalencia del "síndrome de quemarse por el trabajo" (Burnout) en pediatras de hospitales generales. En Revista Saúde Pública, 42, pp. 450-6.

Gil-Monte, P. R. y Peiró, J. (1997). Desgaste Psíquico en el Trabajo: el Síndrome de Quemarse, Madrid: Síntesis Psicológica.

Gil-Monte, P. R. y Peiró, J. (1999). Perspectivas teóricas y modelos interpretativos para el estudio del síndrome de quemarse por el trabajo. En Anales de Psicología, 15, pp. 261-268.

Gil-Monte, P. y Peiró, J. (2000). Un modelo comparativo sobre criterios normativos y diferenciales para el diagnóstico del Síndrome de quemarse por el trabajo (Burnout) según el MBI-HSS. En Revista de Psicología del Trabajo y de las Organizaciones, 16, pp. 135-149.

Guerrero, E. (2003). Análisis pormenorizado de los grados de Burnout y técnicas de afrontamiento del estrés docente en profesorado universitario. En Anales de Psicología, 19, pp. 145-158.

Hernández, L., Olmedo, E. e Ibáñez, I. (2003). Estar quemado y su relación con el afrontamiento. En International Journal of Clinical and Health Psychology, 4, pp. 323-336.

Kuras, S. y Resnizky, S. (2003). Acompañantes terapéuticos: Actualización teóricoclínica, Buenos Aires: Letra Viva.

Lazarus, R. y Folkman, S. (1986). Estrés y procesos cognitivos. Madrid: Ediciones Martínez Roca.

Maslach, C. y Jackson, S. (1997). Manual MBI - Inventario “Burnout” de Maslach. Madrid: TEA Ediciones.

Morales, G., Pérez, J. y Menares, M. (2003). Procesos emocionales de cuidado y riesgo en profesionales que trabajan con el sufrimiento humano. En Revista de Psicología, 12, pp. 9-25.

Neira, C. (2004). Cuando se enferman los que curan. Estrés laboral y Burnout en los profesionales de la salud, Buenos Aires: Gambacop. 
Olivares, V. y Gil-Monte, P. R. (2007). Prevalencia del Síndrome de Quemarse por el trabajo (Burnout) en trabajadores de servicios en Chile. En Información Psicológica, 91, pp. 43-52.

Parris, L. (2007). Estrategias de afrontamiento del estrés asistencial y satisfacción laboral - personal en médicos y enfermeros. En Revista Interamericana de Psicología Ocupacional, 26, pp. 7-21.

Pearlin, L. I. y Schooler, C. (1978). The Structure of Coping. En Journal of Health and Social Behavior, 19, pp. 2-21.

Peinado, A. y Garcés, E. (2004). Burnout en cuidadores principales de pacientes con Alzheimer: El síndrome del asistente desasistido. En Anales de Psicología, 14, pp. 83-93.

Popp, M. (2008). Estudio preliminar sobre el Síndrome de Burnout y estrategias de afrontamiento en enfermeras de unidades de terapia intensiva. En Interdisciplinaria, 25, pp. 5-27.

Robayo y Torres (2002).Exaustão emocional: relaçõescom a percepção de suporte organizacional e com as estratégias de coping no trabalho. En Estudos de Psicología, 7, pp. 37-46.

Sosa, E. N. (2007). Frecuencia de los síntomas del Síndrome de Burnout en profesionales médicos. En Revista Médica de Rosario, 73, pp. 12-20.

Topa, G. y Morales, J. (2007). Burnout e identificación con el grupo: El papel del apoyo social en un modelo de ecuaciones estructurales. En International Journal of Clinical and HealthPsychology, 7, pp. 337-348. 